\title{
Undamaged measurement of the sub-micron diaphragm and gap by tri-beam interference
}

\author{
Yanping Chen ${ }^{1}$, Shen Liu ${ }^{1, *}$, Zhongyuan Sun ${ }^{1,2}$, Lin Zhang ${ }^{2}$, Namita Sahoo ${ }^{2}$, Junxian Luo ${ }^{1}$, Yuanyuan \\ Zhao ${ }^{1}$, Changrui Liao ${ }^{1}$, Bin $\mathrm{Du}^{1}, \mathrm{Chi} \mathrm{Li}^{1}$ and Yiping Wang ${ }^{1}$
}

\begin{abstract}
A simple, high-accuracy and non-destructive method for the measurement of diaphragm thickness and microgap width based on modulated tri-beam interference is demonstrated. With this method, a theoretical estimation error less than $0.5 \%$ for a diaphragm thickness of $\sim 1 \mu \mathrm{m}$ is achievable. Several fiber-tip air bubbles with different diaphragm thicknesses $(6.25,5.0,2.5$ and $1.25 \mu \mathrm{m})$ were fabricated to verify our proposed measurement method. Furthermore, an improved technique was introduced by immersing the measured object into a liquid environment to simplify a four-beam interference into tri-beam one. By applying this improved technique, the diaphragm thickness of a fabricated in-fiber rectangular air bubble is measured to be about $1.47 \mu \mathrm{m}$, and the averaged microgap width of a standard silica capillary is measured to be about $10.07 \mu \mathrm{m}$, giving a corresponding measurement error only $1.27 \%$ compared with actual scanning electron microscope (SEM) results.
\end{abstract}

Index Terms - Diaphragm measurement; microgap measurement; fiber optics communications; tri-beam interference.

\section{INTRODUCTION}

$\mathrm{V}$ ARIOUS types of optical devices with a thin diaphragm have been reported, for example, Fabry-Perot interferometers (FPIs) [1]-[4], whispering-gallery-modes (WGMs) resonator [5-8] and optomechanical cavity [9]-[11]. Such devices are suitable for sensing pressure [1]-[3], [7], acoustics [12], strain [4], and vibration [10], [11]. 1Typically, the WGM resonator [5]-[8], with ultra-thin silica-wall diaphragm and microgap channel, has been developed into an excellent optomechanical sensor that will help unlock vibrational secrets of chemical and biological samples at the nanoscale. This type of resonators is considered as the first-ever bridge between optomechanics and microfluidics, which

1 Manuscript received XXXX, 2019; revised XXXX, 2019; accepted XXXX, 2019. Date of publication XXXX, 2019; date of current version XXXX, 2019.

This work was supported by National Natural Science Foundation of China (grant nos. 61425007, 61635007 and 61405125), Guangdong Natural Science Foundation (grant nos. 2015B010105007, 2014A030308007 and 2015A030313541), Science and Technology Innovation Commission of Shenzhen (grant nos. JCYJ20160427104925452, JCYJ20170412105604705 and JCYJ20170818093743767), and Development and Reform Commission of Shenzhen Municipality foundation.

1. Yanping Chen, Shen Liu, Zhongyuan Sun, Junxian Luo, Yuanyuan Zhao, Changrui Liao, Bin Du, Chi Li and Yiping Wang are affiliated with Key Laboratory of Optoelectronic Devices and Systems of Ministry of Education and Guangdong Province, College of Physics and Optoelectronic Engineering, Shenzhen University, Shenzhen 518060, China.

2. Zhongyuan Sun, Lin Zhang, Namita Sahoo are affiliated with Aston Institute of Photonic Technologies, Aston University, Birmingham, UK

(*Corresponding author e-mail: shenliu@szu.edu.cn) has been used to sense refractive index [13] and detect biomolecules [14]. However, their sensing ability strongly relies on the spatial and temporal confinement of light by the resonator, characterized by its mode volume $\mathrm{V}$ and its quality factor $Q$. These characteristics have a close relationship with the silica-wall diaphragm thickness of the resonator along its equator surface. For a given WGM, an optimized silica-wall diaphragm thickness will significantly enhance the response sensitivity in sensing [15]. As a result, knowing the size of diaphragm thickness and microgap width is important for optimizing sensing applications within specific environments. Generally, the methods of measuring the diaphragm thickness and microgap width are divided into direct and indirect measurement. The former can directly and accurately measure the diaphragm thickness and microgap width, generally by using a scanning electron microscope (SEM) [1] or a transmission electron microscope (TEM) [16]. However, this method not just requires expensive instruments but also destroys the structure of the sample. The indirect measurement has been reported by employing optical techniques, which are non-destructive for measuring diaphragm thickness, such as using the optical microscope [2], prism coupler [17], ellipsometry [18] and Fizeau interferometer [19]. However, these optical methods also need complex optical elements and the calculation for obtaining the diaphragm thickness is more sophisticated.

In this paper, we propose and demonstrate a novel, simple and non-destructive measurement method for measuring diaphragm thickness and microgap width with high accuracy. This method is based on monitoring the free spectral range (FSR) of an envelope generated from tri-beam interference. Firstly, we theoretically derive an analytic mode for an FPI with three reflected surfaces and use it to calculate the diaphragm thickness and microgap width. Then, we demonstrate the experimental measurement employing this tri-beam interference method for several fabricated fiber-tip air bubbles with different diaphragm thicknesses and compare the results with theoretical values and the measurement results using standard SEM. Finally, an improved measurement technique is introduced for measuring two different type samples, i.e. in-fiber rectangle air bubble with a thin diaphragm and silica capillary with a microgap. The corresponding diaphragm thickness and averaged microgap width are measured to be about 1.47 and $10.07 \mu \mathrm{m}$, respectively, and the measurement error is only $1.27 \%$.

\section{ANALYTICAL MODEL AND NUMERICAL SimULATION}

As shown in Fig. 1(a), a typical measurement set-up is employed for monitoring the reflection spectrum of an 


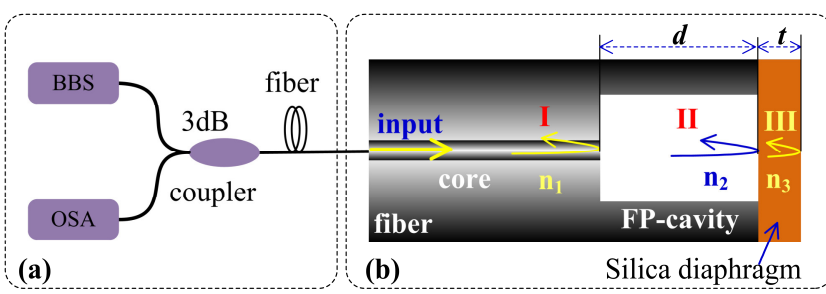

Fig. 1. (a) The corresponding optical measurement set-up. (b) the standard model of tri-beam interference based on in-fiber FPI with silica diaphragm.

in-fiber Fabry-Perot interferometer (FPI), which consists of a $3 \mathrm{~dB}$ fiber coupler, a broad-band light source (BBS), and an optical spectrum analyzer (OSA). This typical measurement set-up is employed in both the simulation and the actual experiment as following discussion. The schematic of in-fiber FPI based on an air-cavity and a silica diaphragm is illustrated in Fig. 1(b), where $n_{1}, n_{2}$, and $n_{3}$ are the effective reflective indexes of fiber-end surface, air-cavity, and silica diaphragm, respectively.

The reflectivity from a silica glass in-fiber FPI is very low, only about $3.5 \%$, so that the high-order reflections of the light in the FP-cavity are negligible. It can be seen clearly in Fig. 1(b), the light from the broad-band light source is launched into the in-fiber FPI, and propagates though the air-cavity and silica diaphragm. Based on the Fresnel equations, the propagating light is reflected on the fiber end surface (I), inner (II) and outer (III) surfaces of the silica diaphragm, respectively. Therefore, the interference spectrum captured by OSA is formed by these three reflected lights. Then, the corresponding interference spectrum can be simply described by a tri-beam interference approximation. So the total reflected intensity can therefore be written as below [20]:

$$
\begin{aligned}
I= & |E|^{2}=\left|E_{1}-E_{2} \exp \left(\frac{4 \pi}{\lambda} n_{\text {air }} d\right)+E_{3} \exp \left[\frac{4 \pi}{\lambda}\left(n_{\text {silica }} t+n_{\text {air }} d\right)\right]\right|^{2} \\
= & \underbrace{E_{1}^{2}+E_{2}^{2}+E_{3}^{2}}_{\mathrm{A}} \underbrace{-2 E_{1} E_{2} \cos \left(\frac{4 \pi}{\lambda} n_{\text {air }} d\right)}_{\mathrm{B}}-\underbrace{2 E_{2} E_{3} \cos \left(\frac{4 \pi}{\lambda} n_{\text {silica }} t\right)}_{\mathrm{C}} \\
& +\underbrace{2 E_{1} E_{3} \cos \left[\frac{4 \pi}{\lambda}\left(n_{\text {silica }} t+n_{\text {air }} d\right)\right]}_{\mathrm{D}}
\end{aligned}
$$

where $E_{1}, E_{2}, E_{3}$ are the amplitudes of reflected light from the three surfaces (I, II, III), $t$ is thickness of the silica diaphragm, $d$ is FPI's air-cavity length, $\lambda$ is an input light wavelength, and $n_{\text {air }}$ and $n_{\text {silica }}$ are the refractive indices of the air and silica, here, $n_{1}=n_{3}=n_{\text {silica }}$, and $n_{2}=n_{\text {air }}$.

Notice that, Eq. (1) includes four terms, and the first term-A is a constant. According to standard two-beam interference [21], the second term-B can be considered as the air-cavity interference between surface-( $\left(I_{1} \mathrm{E}_{1}\right)$ and surface-(II, $\mathrm{E}_{2}$ ), which depends on both the FP-cavity length of $d$ and air refractive index $n_{\text {air }}$. Similarly, the third term-C is the diaphragm interference between surface-(II, $\mathrm{E}_{2}$ ) and surface-(III, $\mathrm{E}_{3}$ ) with an FP-cavity length of $t$ and silica refractive index $n_{\text {silica }}$. For individual interference generated by term-B or term-C, thus, the FSR of the reflection spectrum can thus be calculated by [21]:

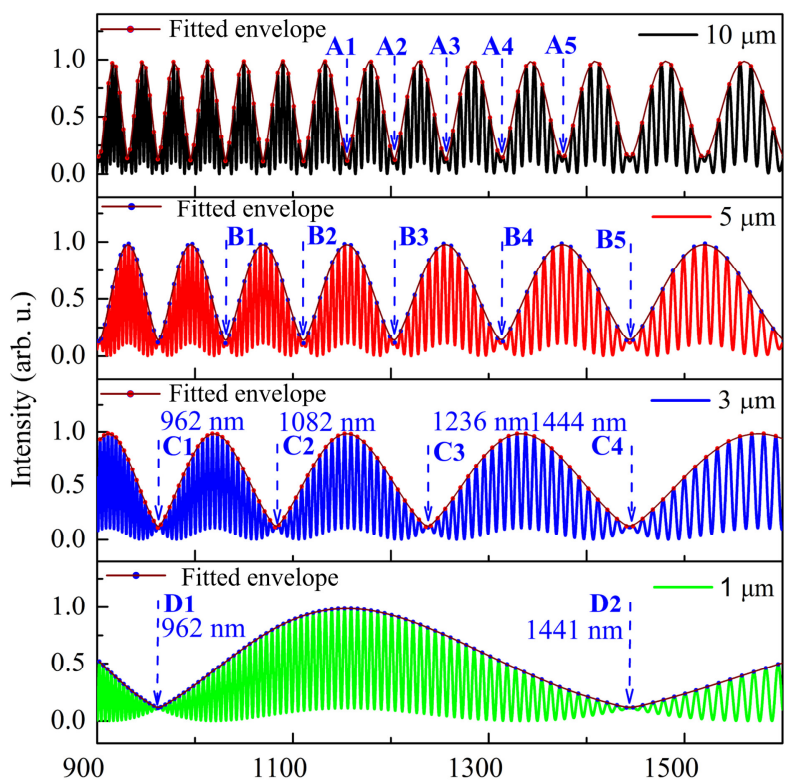

(a) Wavelength (nm)

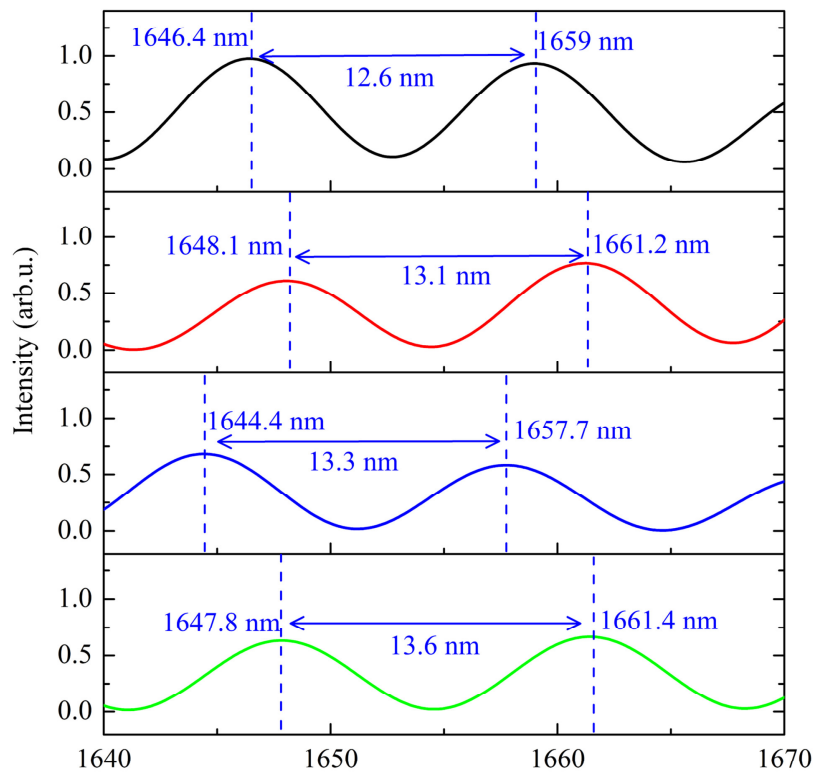

(b) Enlarged view

Fig. 2. (a) The envelope FSR of the interference spectrum is enlarging with a reduced diaphragm thickness. (b) The enlarged view of interference spectrum at the wavelength range from 1640 to $1670 \mathrm{~nm}$.

$$
\operatorname{FSR}_{(m, m-1)}=\left(\frac{\lambda_{m} \lambda_{m-1}}{2 n_{\text {eff }} L_{\mathrm{FP}-\text { cavity }}}\right)
$$

where $\lambda_{m}$ is the resonance wavelength of the $m^{\text {th }}$ order interference dip/peak and $n_{\text {eff }}$ is the effective-index of intra-cavity medium, and $L_{F P \text {-cavity }}$ is the FP-cavity length. However, in Eq. (1), the linear superposition between term- $\mathrm{B}$ and term- $\mathrm{C}$ is modulated by the correlation term- $\mathrm{D}$, which is an associated reflection interference, modulating the entire spectrum with a slowly varying envelope.

To investigate the relationship between envelope and diaphragm thickness, a numerical simulation based on Eq. (1), has been achieved covering the wavelength range from 900 to $1600 \mathrm{~nm}$ with a step of $0.1 \mathrm{~nm}$, and under condition of $n_{\text {silica }}=1.45, n_{\text {air }}=1, d=100 \mu \mathrm{m}$, satisfying $n_{\text {silica }} t<<n_{\text {air }} d$; here, it is assumed that the input light from optical fiber is strictly perpendicular to the parallel reflection surfaces (I, II, III) shown in Fig. 1(b), and the higher-order reflections 
from the reflected surfaces (I, II, III) is ignored due to the small reflectivity of the silica-air interface, only about $3.5 \%$. For simplify, the amplitudes of reflected light from the three surfaces are set as the same value, i.e. $\mathrm{E}_{1}=\mathrm{E}_{2}=\mathrm{E}_{3}$. Thus, the normalized spectra can be calculated and shown in Fig. 2(a), and we can see that the interference spectrum is modulated by a slowly varying envelope (the dotted line), whose FSR changes with the thickness of the silica diaphragm. This envelope is fitted by the MATLAB's toolbox (Fit type: Smoothing Spline, Curve Fitting Tool in MATLAB R2018a), and R-square $\left(R^{2}\right)$ of the curve fitting is selected to describe the goodness of fit [22], where the $\mathrm{R}^{2}$ is calculated to be $0.9927,0.9994,0.9999$, and 1 , describing the goodness of fit of the spectral envelope with diaphragm thickness $10,5,3$, and $1 \mu \mathrm{m}$, respectively. Notice that the estimation errors are closely related to the numerical sampling intervals, which determine the value accuracy of the envelope peak/dip.

In the following discussion, we will analyze the physical significance of Eq. (1) under two limiting mathematical conditions. Here, we assume the diaphragm thickness is very thin, i.e. $t \rightarrow 0$, as the first condition, and satisfying $n_{\text {silica }} t<<n_{\text {air }} d$. Eq. (1) can then be simplified as:

$$
\begin{aligned}
I \approx \underbrace{E_{1}^{2}+E_{2}^{2}+E_{3}^{2}}_{\mathrm{A}}-2 E_{1}\left(E_{2}-E_{3}\right) \underbrace{\cos \left(\frac{4 \pi}{\lambda} n_{\text {air }} d\right)}_{\mathrm{B}^{\prime}} \\
-2 \mathrm{E}_{2} E_{3} \underbrace{\cos \left(\frac{4 \pi}{\lambda} n_{\text {silica }} t\right)}_{\mathrm{C}^{\prime}} \quad(\text { if } t \rightarrow 0)
\end{aligned}
$$

If $t \rightarrow 0$, in Eq. (1), there is almost no phase difference between the B- and D- terms. For Eq. (3), we clearly see that the total reflected interference intensity $I$ is the result of the linear superposition by two interfering wave-functions, i.e. $\mathrm{B}^{\prime}$ and $\mathrm{C}^{\prime}$, corresponding to the air-cavity and diaphragm interference mentioned above. Obviously, the spectral envelope is the diaphragm interference, which slowly modulates the fringe of air-cavity interference spectrum. So, the air-cavity length and diaphragm thickness can be estimated by deriving Eq. (2):

$$
t_{(m, m-1)}=\frac{\lambda_{m} \lambda_{m-1}}{2 n_{\text {silica }} F S R_{\text {envelope }(m, m-1)}}
$$

where, $\lambda_{m}$ is the interference dip of the spectral envelope, and $F S R_{\text {envelope }(m, m-1)}$ is the corresponding length of the spectral envelope. Using Eq. (4) and the simulated results in Fig. 2(a), the diaphragm thickness $t$ can be estimated by a series of envelope FSRs.

As shown in Fig. 2(a), five contiguous resonant dips of envelope, are selected to be $1154,1202,1255,1312$, and $1375 \mathrm{~nm}$, corresponding to the marked points A1, A2, A3, A4 and A5, respectively. The silica diaphragm thicknesses are calculated to be about 9.96, 9.82, 9.96, and $9.87 \mu \mathrm{m}$, comparing to the theoretical diaphragm thickness of $10 \mu \mathrm{m}$, the estimation error is about $0.98 \%$. When the theoretical diaphragm thickness is $5 \mu \mathrm{m}$, we also select five contiguous resonant dips of envelope as 1031, 1111, 1204, 1315 , and $1444 \mathrm{~nm}$, i.e. the wavelength values of marked points B1, B2, B3, B4 and B5, respectively. Four silica diaphragm thicknesses $(4.94,4.96,4.92$, and $5.08 \mu \mathrm{m})$ can be calculated approximately by means of Eq. (4), and the estimation error is about $0.50 \%$. Similarly, we calculate the estimation error to be about $0.67 \%$ and $0.20 \%$ relative to the theoretical diaphragm thicknesses 3 and $1 \mu \mathrm{m}$, respectively. Thus, we clearly see that the envelope FSR of the interference spectrum increases with the decreases of the diaphragm thickness, and the silica diaphragm thickness can be estimated by means of Eq. (4), where the estimation accuracy increases with the silica diaphragm thickness decreasing.

Analogously, an enlarged view of the interference spectrum with wavelength range from 1640 to $1670 \mathrm{~nm}$ is shown in Fig. 2(b). From these zoomed figures, it can be seen that the FSR of the FPI interference spectrum also gradually increases with the decreases of the diaphragm thickness, as we see the simulated FSR values for the FPI interference at around $1655 \mathrm{~nm}$ are 12.6, 13.1, 13.3, and $13.6 \mathrm{~nm}$, respectively. Similarly, combining with the results of Fig. 2(b), the estimated air-cavity lengths are calculated to be $108.39,104.50,102.48$, and $100.65 \mu \mathrm{m}$, respectively. Comparing with the theoretical value of air-cavity length $100 \mu \mathrm{m}$, the corresponding estimated errors are $8.39 \%, 4.5 \%, 2.48 \%$, and $0.65 \%$, respectively. Thus, the approximation of Eq. (3) is reasonable under the first assumed condition (i.e. $t \rightarrow 0$ ). Therefore, we can optically measure the thickness of silica diaphragm using Eq. (4) and the slow-varying envelope FSR when the silica diaphragm is very thin.

For the second condition, assuming the FPI air-cavity length is very short, i.e. $d \rightarrow 0$, which can be regarded as a microgap, which satisfies $n_{\text {air }} d<<n_{\text {silica }} t$. Eq. (1) can then be simplified into:

$$
\begin{aligned}
I \approx \underbrace{E_{1}^{2}+E_{2}^{2}+E_{3}^{2}}_{\mathrm{A}}-2 E_{3}\left(E_{2}-E_{1}\right) \underbrace{\cos \left(\frac{4 \pi}{\lambda} n_{\text {silica }} t\right)}_{\mathrm{C}^{\prime}} \\
-2 E_{1} \underbrace{}_{\mathrm{B}_{2}} \underbrace{\cos }_{\text {'os }\left(\frac{4 \pi}{\lambda} n_{\text {air }} d\right)}
\end{aligned}
$$

As in the analysis of Eq. (4), the modulation of the fringe envelope is regarded as the microgap interference, and the microgap width $d$ can be calculated from:

$$
d_{(m, m-1)}=\frac{\lambda_{m} \lambda_{m-1}}{2 n_{\text {air }} F S R_{\text {envelope }(m, m-1)}}
$$

In other word, using Eq. (4) and Eq. (6), we can estimate the diaphragm thickness and microgap width of the FPI system by monitoring the envelope FSR of the tri-beam interference spectrum, where the two mathematical conditions $t \rightarrow 0$ and $d \rightarrow 0$ are satisfied respectively.

\section{MATERIALS AND METHODS}

Fig. 3(a) illustrates a standard model of tri-beam interference based on in-fiber FPI with silica diaphragm; however, it is difficult to fabricate this standard model in-fiber. In the following discussion, an approximate model, i.e. fiber-tip air bubble, is fabricated for verifying the method of diaphragm measurement based on the tri-beam interference. As shown in Fig. 3(b), the fiber-tip air bubble has three reflected surfaces, inner surface-I of the air 


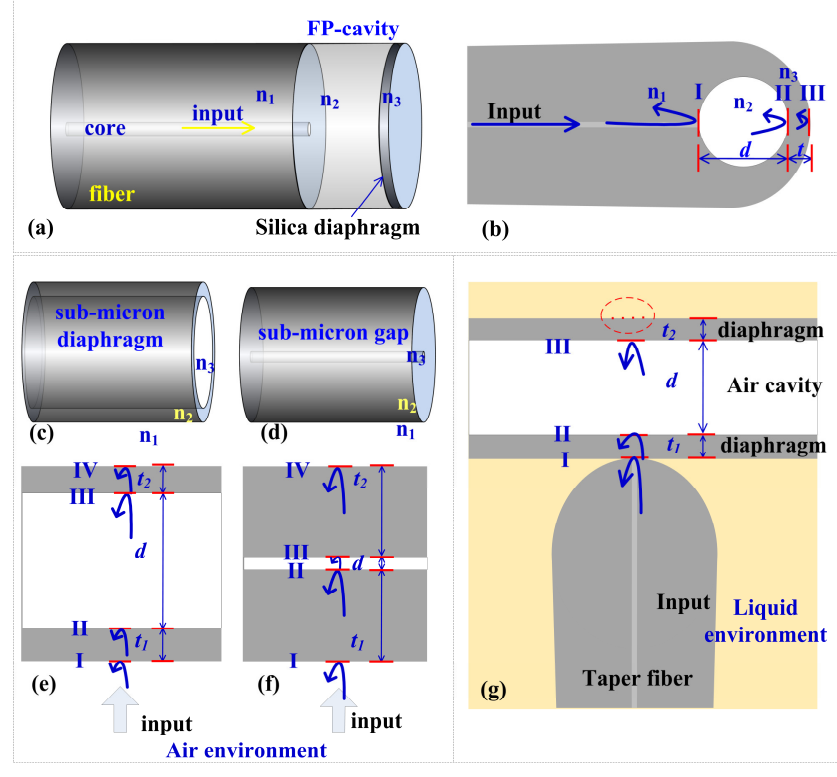

Fig. 3. The schematic of (a) standard tri-beam interference and (b) in-fiber air bubble. (c) shell-shaped structures with silica-wall diaphragm and (d) standard silica capillary with microgap, corresponding to the schematic of four-beam interference (e) and (f) in air environment. (g) The schematic of the simplified tri-beam interference by immersing in a liquid environment.

bubble, inner surface-II and outer surface-III of the silica-wall diaphragm, which will generate a tri-beam interference.

Unlike the fiber-tip air bubble discussed above, as shown in Fig. 3(c), a variety of shell-shaped structures with silica-wall diaphragm do not satisfy the conditions of the standard tri-beam interference, for example, micro-bottle [24], micro-tube [25] or micro-bubble [5], [26]. In air environment, they generally generate four-beam interference, as shown in Fig. 3(e), due to the reflection coming from the double-layer surface of the silica-shell diaphragm, i.e. the reflected surface I, II, III, and IV, respectively. Similarly, as shown in Fig. 3(d), the structures with micro-gap also generate four-beam interference while the input light is strictly perpendicular to the reflection surfaces illustrated in Fig. 3(f). However, the four-beam interference spectrum has no obvious regularity and spectral envelope. Therefore, the sub-micron diaphragm thickness or the sub-micron gap width cannot be calculated by Eq. (4).

In order to measure the diaphragm thickness or the gap width by means of the method introduced in Section II, the four-beam interference may be simplified into a tri-beam interference by a simple technique to eliminate the reflected surface (IV). Taking the diaphragm measurement as an example illustrated in Fig. 3(g), this can be implemented by immersing the shell-shaped structures into a liquid environment, such as water, paraffin oil, or commercial refractive index (RI) matching liquid. As a result, there will be no reflection from the top surface (IV) as the wall thickness on the top can be regarded as infinite when immersed in liquid. In the actual experiment, the shell-shaped structure is demonstrated by using an in-fiber rectangular air bubble with a silica-wall diaphragm, a taper-fiber probe coupling input light is fabricated by a fusion splitter and fixed on a translation stage to control the probe position.

Notice that (1) in our experiments, as shown in Fig. 3(g), a taper fiber with a hemispherical and smooth end is employed as a probe. There are three purposes for employing the taper fiber, (a) it is used to couple the light into the silica-wall surface of shell-shaped structures along its orthogonal direction, (b) the two reflected light mode fields from the end of the taper fiber and the wall surface of shell-shaped structures, are matched with each other due to their similar circular-arc surface, (c) the taper fiber is more easily positioned at the measurement point in the wall surface of the shell-shaped structures. (2) In the actual experiment, the measurement errors should be taken into account in the experiment, i.e. the errors mainly induced by the setting step of OSA, normal incidence or not and the curve fit for approaching resolving the center points of the envelope. For example, the best resolution of the employed OSA is $0.02 \mathrm{~nm}$, which induces an envelope FSR error of $0.04 \mathrm{~nm}$; furthermore, if the input light incidents not in the normal direction, the measurement accuracy of the diaphragm and microgap would be decreased sharply.

\section{EXPERIMENTAL RESULTS}

\section{A. Tri-beam Interference}

In order to demonstrate the tri-beam interference, as shown in Fig. 4, a series of fiber-tip air bubbles, with different silica diaphragm thicknesses were fabricated by means of the improved electrical arc discharge technique, whose details are described in Ref. [23]. Fig. 4(b) shows the microscope images of the fabricated fiber-tip air bubbles, by use of a microscope objective $(20 \times, \mathrm{NA}=0.4)$. The reflection spectra of FPIs based on fiber-tip air bubbles of different silica diaphragm thicknesses are measured by using the typical measurement set-up shown in Fig. 1, and a broad-band light source covering a range from 400 to $2400 \mathrm{~nm}$ and OSA with scanning range from 600 to 1700 $\mathrm{nm}$ are employed. As shown in Fig. 4(a), we can clearly see the interferences are modulated by a slowly varying envelop due to tri-beam interference effect.
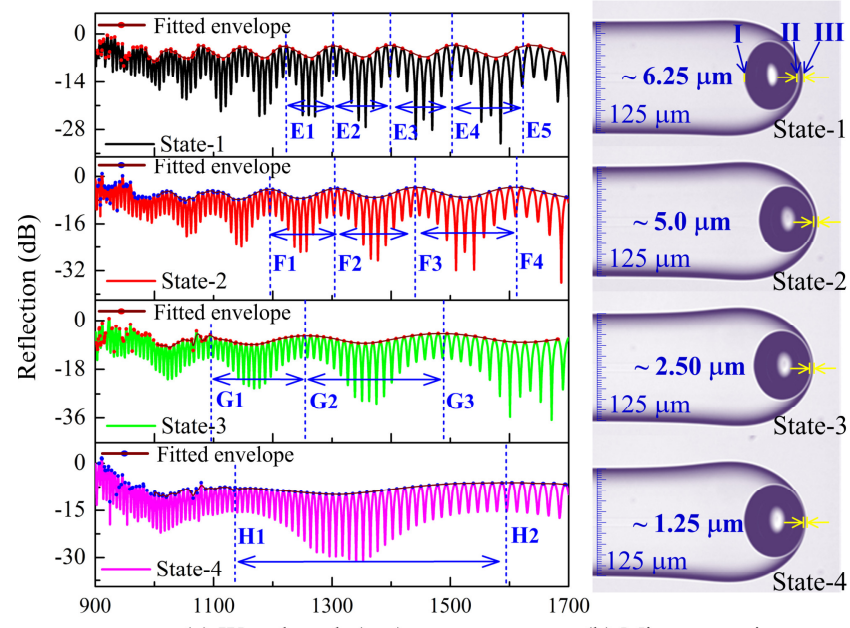

(a) Wavelength (nm)

(b) Microscope images

Fig. 4. (a) The measured interference spectra of fiber-tip air bubbles with different diaphragm thicknesses, showing modulated slow varying envelope. (b) The $20 \times$ microscope images of fiber-tip air bubbles with measured wall thickness. 
As shown in Fig. 4(a), in the state-1, we have fitted the experimental results and the resonant peaks (marked points, E1, E2, E3, E4, E5) of the spectral envelope are obtained as to be $1219,1302,1397,1508$, and $1636 \mathrm{~nm}$. Using Eq. (4), the silica diaphragm thickness is estimated successively to be $6.59,6.60,6.54$, and $6.65 \mu \mathrm{m}$, comparing to the measured diaphragm thickness of 6.25 $\mu \mathrm{m}$ from microscope images, the measurement error is about $5.0 \%$. In the state-2, we also select contiguous resonant peaks (marked points, F1, F2, F3, F4) of the spectral envelope as $1195,1305,1441$, and $1611 \mathrm{~nm}$, respectively. So the silica diaphragm thicknesses can be calculated approximately to be $4.89,4.80$, and $4.71 \mu \mathrm{m}$ by means of Eq. (4), and the measurement error is about $4 \%$. Similarly, in the state-3, the resonant peaks (marked points, $\mathrm{G} 1, \mathrm{G} 2, \mathrm{G} 3$ ) of the spectral envelope is 1090,1259 , and $1489 \mathrm{~nm}$, and the silica diaphragm thickness is calculated to be 2.80 and $2.81 \mu \mathrm{m}$, which are close to the measured diaphragm thickness of $2.5 \mu \mathrm{m}$. In the state-4, there is only one complete FSR of the spectral envelope. The resonant peaks are fitted to be 1140 and $1595 \mathrm{~nm}$, which are used for calculating the silica diaphragm thickness $1.38 \mu \mathrm{m}$. Comparing to the measured value of $1.25 \mu \mathrm{m}$, the measurement error is only $0.13 \mu \mathrm{m}$. Therefore, it clearly showing the effectiveness of the tri-beam interference method to measure the thickness of the diaphragm in the fiber-tip air bubble and the thickness error is less than 1 $\mu \mathrm{m}$.

It is noteworthy that the measured spectra in Fig. 4(a) is different from the simulated spectra in Fig. 2(a), here, main reasons can be reduced to two parts that (1) As shown in Fig. 2(a) simulated by Eq.(1), there is an ideal assumption of amplitudes $E_{1}=E_{2}=E_{3}$, however, in the actual experiment, the three reflected surfaces of the fiber-tip air bubble are not strictly parallel and non-uniform thickness, which leads the actual values of $E_{2}$ and $E_{3}$ to be much smaller than $E_{1}$; (2) The numerical values of the simulation shown in Fig. 2(a) are normalized and absolute value, however, in reality, the measured spectra of the fiber-tip air bubble are the reflected light relative to the well-cleaved fiber end. Furthermore, the measurement errors of the microscope images depend on the resolution $(\sim 0.25 \mu \mathrm{m})$ of optical microscope with the microscope objective $(20 \times, \mathrm{NA}=0.4)$.

\section{B. Diaphragm Thickness Measurement}

As an example, we will here demonstrate how to measure the silica-shell diaphragm thickness of an in-fiber rectangular air bubble reported in Ref. [25]. In Fig. 5(a), the microscope image shows an actual structure of the in-fiber rectangular air bubble and the taper fiber probe with a hemispherical and smooth end. In air environment, the rectangular air bubble generate four-beam interference due to the reflection coming from the double-layer surface of the silica-wall diaphragm (schematic shown in Fig. 3(e)); and the overall reflection spectrum is measured covering wavelength range from 900 to $1700 \mathrm{~nm}$, as shown in Fig. 5(d). However, the four-beam interference spectrum has no obvious regularity and spectral envelope. Therefore, the wall thickness of the rectangular air bubble cannot be calculated by using Eq. (4).
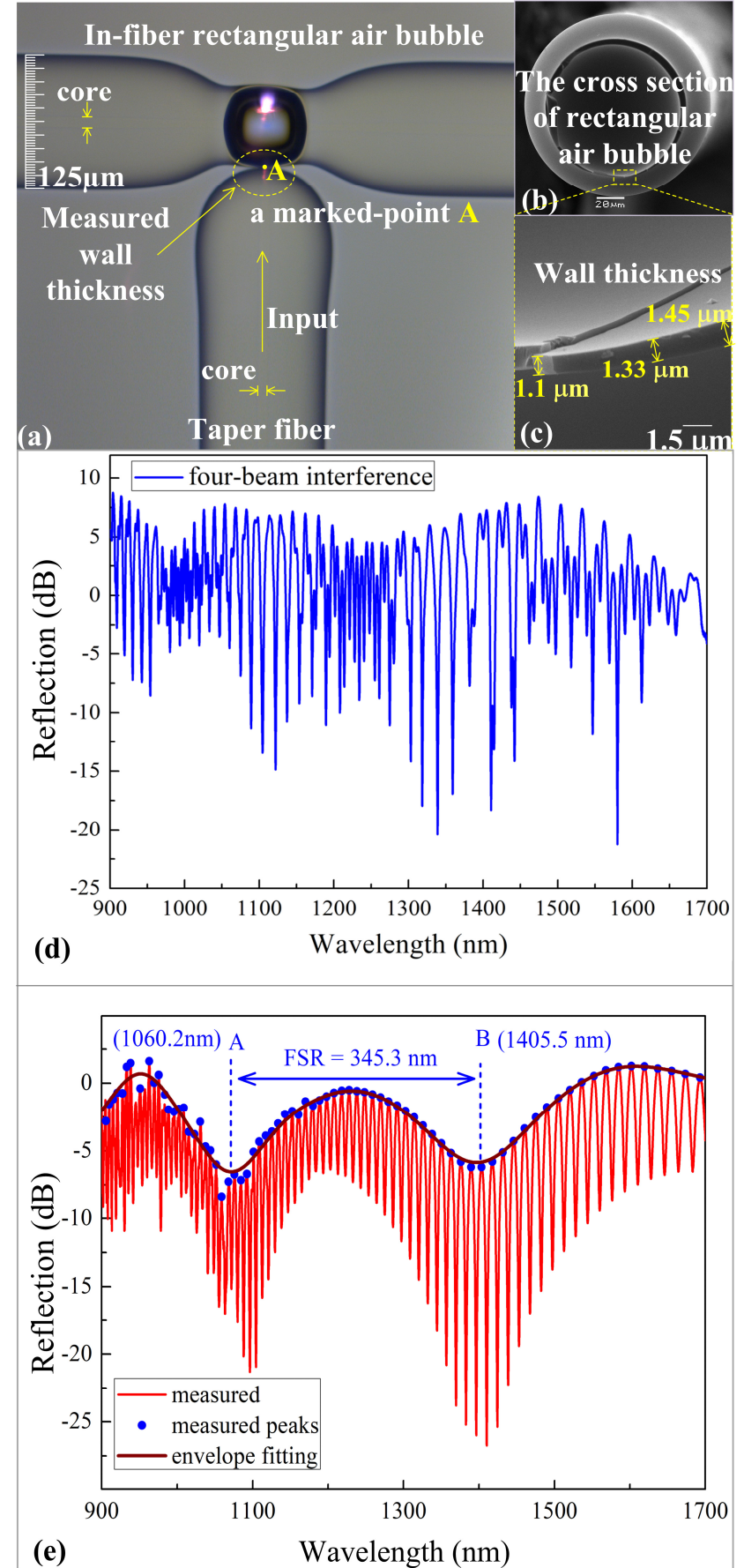

Fig. 5 (a) Microscope image and (b) SEM image of the in-fiber rectangular air bubble. (c) Enlarged partial view of the wall silica-diaphragm. (d) Four-beam interference spectra. (e) The measured interference spectrum with a slow varying envelope.

To satisfy the conditions of the standard tri-beam interference, as shown in Fig. 5(a), both in-fiber rectangular air bubble and taper-fiber probe are immersed into water. Fig. 5(e) illuminates a typical reflection spectrum, clearly exhibiting the tri-beam interference pattern with a slow varying envelope, and the envelope FSR (one envelope length) is about $345.3 \mathrm{~nm}$ with two dips of about 1060.2 and $1405.5 \mathrm{~nm}$, respectively. Thus, the wall thickness of in-fiber rectangular air bubble at the marked-point " $\mathrm{A}$ " is calculated about $1.47 \mu \mathrm{m}$ by using Eq. (4).

In order to prove that our calculated result matches the actual wall thickness of the in-fiber rectangular air bubble, which is cut open along its equatorial direction by a femtosecond laser and measured by an SEM system. The 
SEM image of the cross section of the in-fiber rectangular air bubble is shown in Fig. 5(b). An enlarged partial view of the silica-wall diaphragm is shown in Fig. 5(c), where the wall thickness is measured at different positions to be $1.10,1.33$, and $1.45 \mu \mathrm{m}$, respectively. Although the silica wall has no uniform thickness along its equatorial plane, the values are very close to the calculated value of $1.47 \mu \mathrm{m}$ at the marked-point " $A$ " using the tri-beam interference envelope method.

\section{Microgap Measurement}

If one satisfies the second mathematical condition of $\mathrm{d} \rightarrow 0$ and $n_{\text {air }} d<<n_{\text {silica }} t$, by using Eq.(6), the width of a microgap may also be measured using the tri-beam interference method. As shown in Fig. 6(a), a standard silica capillary with an inner diameter of $10 \mu \mathrm{m}$ (https://www.molex.com) is used to validate the measure-
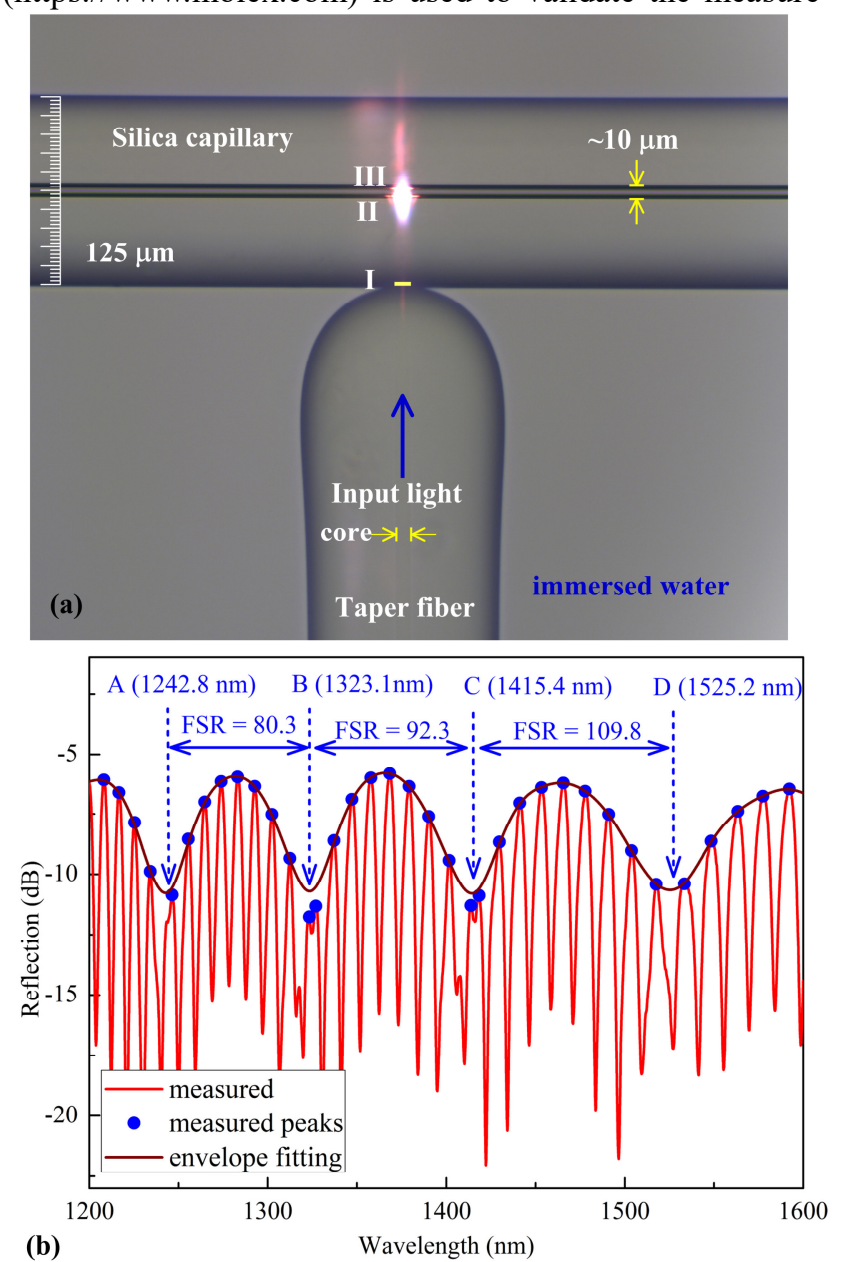

(b)

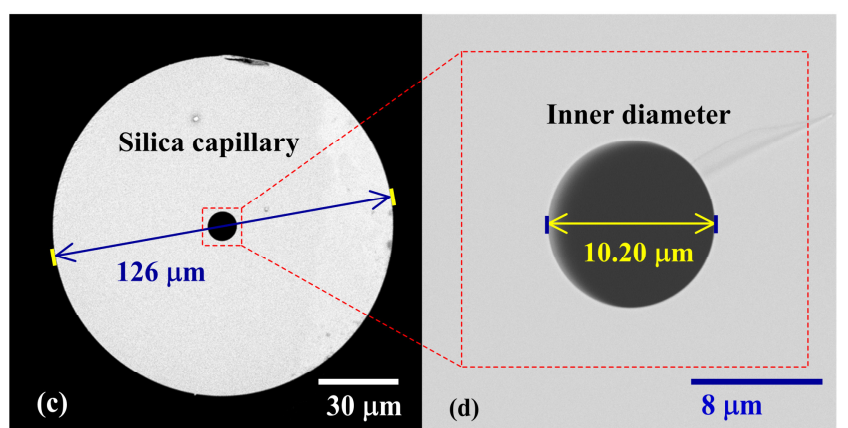

Fig. 6 (a) The optical microscope image of a silica capillary immersed in water environment. (b) The measured interference spectrum and fitted spectral envelope. (c) SEM image of the cross section of silica capillary. (d) Enlarged partial view of the inner diameter. ment method based on tri-beam interference.

To reduce the four-beam reflection to a tri-beam one, the capillary is immersed in water environment. Similar to measure the diaphragm thickness of the in-fiber rectangular air bubble, here, the corresponding reflection spectrum is measured by the OSA with a scanning wavelength from 1200 to $1600 \mathrm{~nm}$, and a slowly varying envelope is evidence as clearly shown in Fig. 6(b). Three FSRs of spectral envelope are measured as $80.3,92.3$, and $109.8 \mathrm{~nm}$. Employing Eq. (6), the widths of the microgap (i.e. the inner diameter of the air-cavity of the silica capillary) are calculated to be $10.24,10.14$, and $9.83 \mu \mathrm{m}$, respectively. As a result, the averaged value of the microgap of the silica capillary is about $10.07 \mu \mathrm{m}$.

Similarly, in order to examine this calculation result, the silica capillary is cut by an optical fiber cleaver and measured using an SEM image. Fig. 6(c) shows the cross section of the silica capillary, for which the corresponding outer diameter is about $126 \mu \mathrm{m}$. An enlarged partial view of the inner diameter (microgap) shows it to be about 10.20 $\mu \mathrm{m}$ in Fig. 6(d). Comparing with the averaged value of $10.07 \mu \mathrm{m}$ above, the measurement error by using Eq. (6) is only about $1.27 \%$. As a result, the obvious advantages of the tri-beam interference method are proved to be low cost, undamaged, and high measurement accuracy, comparing with the methods reported, i.e. SEM [1] or a TEM [16]. Notice that the measurement errors including measurement accuracy of the OSA, noise levels in the system, the curve fit for approaching resolving the center points of the envelope, etc. For example, the minimum resolution of the OSA we used is $0.02 \mathrm{~nm}$, which adds a possible error.

\section{Conclusion}

For estimating diaphragm thickness and microgap width in the micron range, we have demonstrated a simple, high accuracy and non-destructive measurement method, which is based on a tri-beam interference effect. The analytic model has been developed for two limiting conditions, where the thickness of the silica diaphragm and the FPI's air-cavity length are about submicron. In order to validate the tri-beam interference method, the test samples (fiber-tip air bubble, in-fiber rectangular air bubble and a silica capillary) were measured by the microscope and SEM images, and the values from the tri-beam interference method agree well with the microscope and SEM results with measurement errors less than $1.27 \%$. It is foreseeing this simple, high accuracy and non-destructive tri-beam interference method can be used for many other types of optical diaphragms and microgaps thickness determining, detecting crystal cracks and as well as measuring many other microstructures in real applications.

\section{REFERENCES}

[1]. S. Liu, Y. Wang, C. Liao, J. He, C. Fu, K. Yang, Z. Bai, and F. Zhang, "Nano silica diaphragm in-fiber cavity for gas pressure measurement," Sci. Rep., vol. 7, no. 1, 2017.

[2]. F. Xu, D. Ren, X. Shi, C. Li, W. Lu, L. Lu, L. Lu, and B. Yu, "High-sensitivity Fabry-Perot interferometric pressure sensor based on a nanothick silver diaphragm," Opt. Lett., vol. 37, no. 2, pp. 133-135, 2012.

[3]. J. Ma, W. Jin, H. L. Ho, and J. Y. Dai, "High-sensitivity fiber-tip 
pressure sensor with graphene diaphragm," Opt. Lett., vol. 37, no. 13, pp. 2493-2495, 2012

[4]. S. Liu, K. Yang, Y. Wang, J. Qu, C. Liao, J. He, Z. Li, G. Yin, B. Sun, and J. Zhou, "High-sensitivity strain sensor based on in-fiber rectangular air bubble," Sci. Rep., vol. 5, pp. 7624, 2015.

[5]. M. Sumetsky, "Optical microbubble resonator," Opt. Lett., vol. 35, no. 7, pp. 898-900, 2010.

[6]. M. Pöllinger, D. O'Shea, F. Warken, and A. Rauschenbeutel, "Ultra-high-Q tunable whispering-gallery-mode microresonator," Phys. Rev. Lett.,vol. 103, no.5, pp. 053901, 2009.

[7]. R. Henze, T. Seifert, J. Ward, and O. Benson, "Tuning whispering gallery modes using internal aerostatic pressure," Opt. Lett., vol. 36, no. 23 pp. 4536-4538, 2011.

[8]. K. H. Kim, G. Bahl, W. Lee, J. Liu, M. Tomes, X. Fan, and T. Carmon, "Cavity optomechanics on a microfluidic resonator with water and viscous liquids," Light-Sci. Appl., vol. 2, no.11, pp. e110, 2012.

[9]. A. M. Jayich, J. C. Sankey, B. M. Zwickl, C. Yang, J. D. Thompson, S. M. Girvin, A. A. Clerk, F. Marquardt, and J. G. E. Harris, "Dispersive optomechanics: a membrane inside a cavity," New J. Phys.,vol. 10, no.9, pp. 28, 2008.

[10]. N. E. Flowersjacobs, S. W. Hoch, J. C. Sankey, A. Kashkanova, A. M. Jayich, C. Deutsch, J. Reichel, and J. G. E. Harris, "Fiber-cavity-based optomechanical device," Appl. Phys. Lett., vol. 101, no.22, pp. 1693, 2012

[11]. Y. Dhayalan, I. Baskin, K. Shlomi, and E. Buks, "Phase Space Distribution Near the Self-Excited Oscillation Threshold," Phys. Rev. Lett., vol. 112, no.112, pp. 210403, 2014.

[12]. J. Ma, H. Xuan, H. L. Ho, W. Jin, Y. Yang, and S. Fan, "Fiber-Optic Fabry-Pérot Acoustic Sensor With Multilayer Graphene Diaphragm," IEEE Photonics Technol. Lett., vol. 25, no.10, pp. 932-935, 2013

[13]. S. Berneschi, D. Farnesi, F. Cosi, G. N. Conti, S. Pelli, G. C. Righini, and S. Soria, "High Q silica microbubble resonators fabricated by arc discharge," Opt. Lett., vol. 36, no.17, pp. 3521-3523, 2011.

[14]. X. Zhang, L. Liu, and L. Xu, "Ultralow sensing limit in optofluidic micro-bottle resonator biosensor by self-referenced differential-mode detection scheme," Appl. Phys. Lett., vol. 104, no.3, pp. 22242, 2014

[15]. G. Huang, V. A. BolaÃ \pm os Qui $\tilde{A} \pm$ ones, F. Ding, S. Kiravittaya, Y. Mei, and O. G. Schmidt, "Rolled-up optical microcavities with subwavelength wall thicknesses for enhanced liquid sensing applications," Acs Nano, vol. 4, no.6, pp. 3123-3130, 2010.

[16]. S. J. Pennycook and L. A. Boatner, "Chemically sensitive structure-imaging with a scanning transmission electron microscope," Nature, vol. 336, no.6199, pp. 565-567, 1988.

[17]. R. Ulrich and R. Torge, "Measurement of Thin Film Parameters with a Prism Coupler," Appl. Opt., vol. 12, pp. 2901-8, 1973

[18]. C. Y. Han, Z. Y. Lee, and Y. F. Chao, "Determining thickness of films on a curved substrate by use of ellipsometric measurements," Appl. Opt., vol. 48,no.12, pp. 3139, 2009.

[19]. B. F. Oreb, J. Burke, K. Hibino, and P. S. Fairman, "Simultaneous measurement of surface shape and variation in optical thickness of a transparent parallel plate in wavelength-scanning Fizeau interferometer," Appl. Opt., vol. 43,no.6, pp. 1241-9, 2004.

[20]. Y. Zhu, K. L. Cooper, G. R. Pickrell, and A. Wang, "High-Temperature Fiber-Tip Pressure Sensor," J. Lightwave Technol., vol. 24, no.2,pp. 861-869, 2006.

[21]. S. Liu, Y. Wang, C. Liao, G. Wang, Z. Li, Q. Wang, J. Zhou, K. Yang, X. Zhong, and J. Zhao, "High-sensitivity strain sensor based on in-fiber improved Fabry-Perot interferometer," Opt. Lett., vol. 39, no.7, pp. 2121-4, 2014

[22]. J. Chen, "Testing goodness of fit of polynomial models via spline smoothing techniques," Statist. Probab. Lett., vol. 19, no.1, pp. 65-76, 1994

[23]. C. Liao, S. Liu, L. Xu, C. Wang, Y. Wang, Z. Li, Q. Wang, and D. N. Wang, "Sub-micron silica diaphragm-based fiber-tip Fabry-Perot interferometer for pressure measurement," Opt. Lett., vol. 39, no.10, pp. 2827-2830, 2014.

[24]. H. Li, Y. Guo, Y. Sun, K. Reddy, and X. Fan, "Analysis of single nanoparticle detection by using 3-dimensionally confined optofluidic ring resonators," Opt. Express, vol. 18, no.24, pp. 25081-25088, 2015.

[25]. S. Li, L. Ma, H. Zhen, M. R. Jorgensen, S. Kiravittaya, and O. G. Schmidt, "Dynamic axial mode tuning in a rolled-up optical microcavity," Appl. Phys. Lett., vol. 101, no.23,pp. 839, 2012.

[26]. S. Liu, Z. Sun, L. Zhang, C. Fu, Y. Liu, C. Liao, J. He, Z. Bai, Y. Wang, and Y. Wang. "Strain-based tunable optical microresonator with an in-fiber rectangular air bubble," Opt. Lett., vol. 43, no.17, pp. 4077-4080, 2018.
Yanping Chen was born in Chongqing, China, in 1990. She received the M.S. degree in optical engineering from Chongqing University of Posts and Telecommunications in 2016. She is currently pursuing the Ph.D. degree in optical engineering at Shenzhen University.

Shen Liu was born in Henan, China, in 1986. He received the B.Eng. degree in electronic and information engineering from PLA Air Force No.1 Aviation University in 2008, the M.S. degree in circuit and system from Chongqing University of Posts and Telecommunications in 2013, and the Ph.D. degree in optics in Shenzhen University in 2017. From 2017 to 2018, he was with Aston University, Birmingham, UK, as a Postdoctoral Fellow. Since Sept., 2018, he has been in Shenzhen University, Shenzhen, China, as an Assistant Professor. His current research interests focus on optical fiber sensors, WGMs resonator, and cavity optomechanics. He has authored or coauthored 11 patent applications and more than 30 journal and conference papers. ( liushen.szu@gmail.com )

Zhongyuan Sun received the B.Eng. degree from the Tianjin University of Technology and Education, Tianjin, China, in 2007, and the M.S. degree from Edinburgh Napier University, U.K., in 2009, and the Ph.D. degree in photonics from the Aston Institute of Photonic Technologies (AIPT), Aston University, U.K., in 2016. He has been a post-doc Research Fellow, Shenzhen University. $\mathrm{He}$ has been investigating the design and fabrication of novel fiber grating devices and their applications in sensor devices and fiber laser systems.

Changrui Liao was born in Shandong, China, in 1984. He received the B.Eng. degree in optical engineering and the M.S. degree in physical electronics from Huazhong University of Science and Technology (HUST), Wuhan, China, in 2005 and 2007, respectively, and the Ph.D. degree in electrical engineering from the Hong Kong Polytechnic University, Hong Kong, in 2012. He is currently with Shenzhen University, Shenzhen, China, as an Associate Professor. His current research interests focus on femtosecond laser micromachining, optical fiber sensors, and opto microfluidics.

Lin Zhang, received Ph.D. in 1990 from Sussex University, UK, and pursued a post-doc there from 1991 to 1994, and then joined the Photonics Research Group (now Aston Institute of Photonic Technologies) at Aston University, UK. She took the academic position as a lecturer in the School of Engineering \& Applied Science (SEAS), Aston University, UK in 2001 and promoted to a chair professor in 2005. Her research expertise area is in photonics and specialized in optical fiber grating devices and systems, technologies and applications. So far she has authorship to 450 papers and 3 books.

Namita Sahoo received her M.Tech and B.Tech in Optics and Optoelectronics from University of Calcutta, India. She worked as a senior research fellow at the same university for 2 years in the area of Raman effect in Fiber and it's application for signal amplification. She pursued a research on Erbium doped fiber amplier (EDFA) in multichannel amplification from Central Glass and Ceramic Research Institute (CGCRI) in Kolkata, India as a part of her Master's degree project. She is currently pursuing her $\mathrm{PhD}$ in Aston University, UK and her research is focused on Fiber Bragg grating sensors and it's Application in sensing. 
Junxian Luo was born in Guangxi, China, in 1996. She received the B.S. degree in Optoelectronic Information Science Technology and Engineering from Guilin University of Electronic Technology, in 2018. Her current research interests focus on optical fiber resonator and phase-shifted Bragg grating based on femtosecond laser micro-processing technology.

Yuanyuan Zhao was born in Jilin, China, in 1996. She received the B.S. degree in Electronic Science and Technology from Changchun University of Science and Technology, in 2018. Her current research interests focus on design and fabrication of novel fiber grating devices and their applications in sensor devices.

Bin Du was born in Henan China, in 1992. He received the B.S. degree in Flight Vehicle Propulsion Engineering in School of Astronautics from Beihang University, in 2016. His current research interests focus on fiber vibration and ultrasound sensor.

Chi Li was born in Hunan, China, in 1995. He received the B.S. degree in Optoelectronic Information Science Technology and Engineering from Dalian Maritime University, in 2017. His current research interests focus on fiber-integrated polymer functional nano/micro-structure based on fentosecond laser indeced multi-photon polmerization technology.

Yiping Wang (SM'11) was born in Chongqing, China, in 1971. He received the B.Eng. degree in precision instrument engineering from Xi'an Institute of Technology, China, in 1995 and the M.S. degree and Ph.D. degree in optical engineering from Chongqing University, China, in 2000 and 2003, respectively. From 2003 to 2005, he was with Shanghai Jiao Tong University, China, as a Postdoctoral Fellow. From 2005 to 2007, he was with the Hong Kong Polytechnic University, as a Postdoctoral Fellow. From 2007 to 2009, he was with the Institute of Photonic Technology (IPHT), Jena, Germany, as a Humboldt Research Fellow. From 2009 to 2011, he was with the Optoelectronics Research Centre (ORC), University of Southampton, U.K., as a Marie Curie Fellow. Since 2012, he has been with Shenzhen University, Shenzhen, China, as a Distinguished Professor. His current research interests focus on optical fiber sensors, fiber gratings, and photonic crystal fibers. He has authored or coauthored 1 book, 21 patent applications, and more than 240 journal and conference papers. Prof. Wang is a Senior Member of IEEE, the Optical Society of America, and the Chinese Optical Society. 